\title{
Simple Measures of Function and Symptoms in Hospitalized Heart Failure Patients Predict Short-Term Cardiac Event-Free Survival
}

\author{
Evanthia Zaharias, ${ }^{1}$ Janine Cataldo, ${ }^{2}$ Lynda Mackin, ${ }^{3}$ and Jill Howie-Esquivel ${ }^{2}$ \\ ${ }^{1}$ Department of Case Management, University of California, San Francisco Medical Center, 505 Parnassus Avenue, \\ San Francisco, CA 94143-0208, USA \\ ${ }^{2}$ Department of Physiological Nursing, University of California, San Francisco, 2 Koret Way, San Francisco, CA 94143-0610, USA \\ ${ }^{3}$ Department of Physiological Nursing, University of California, 2 Koret Way, San Francisco, CA 94143-0610, USA
}

Correspondence should be addressed to Evanthia Zaharias; evanthia.zaharias@ucsf.edu

Received 6 November 2013; Accepted 19 December 2013; Published 9 February 2014

Academic Editor: Kathleen Finlayson

Copyright (C) 2014 Evanthia Zaharias et al. This is an open access article distributed under the Creative Commons Attribution License, which permits unrestricted use, distribution, and reproduction in any medium, provided the original work is properly cited.

Background. Heart failure (HF) is a prevalent chronic condition where patients experience numerous uncomfortable symptoms, low functional status, and high mortality rates. Objective. To determine whether function and/or symptoms predict cardiac event-free survival in hospitalized HF patients within 90 days of hospital discharge. Methods. Inpatients $(N=32)$ had HF symptoms assessed with 4 yes/no questions. Function was determined with NYHA Classification, Katz Index of Activities of Daily Living (ADLs), and directly with the short physical performance battery (SPPB). Survival was analyzed with time to the first postdischarge cardiac event with events defined as cardiac rehospitalization, heart transplantation, or death. Results. Mean age was $58.2 \pm 13.6$ years. Patient reported ADL function was nearly independent (5.6 \pm 1.1$)$ while direct measure (SPPB) showed moderate functional limitation (6.4 \pm 3.1$)$. Within 90 days, $40.6 \%$ patients had a cardiac event. At discharge, each increase in NYHA Classification was associated with a 3.4-fold higher risk of cardiac events (95\% CI 1.4-8.5). Patients reporting symptoms of dyspnea, fatigue, and orthopnea before discharge had a 4.0 -fold, 9.7 -fold, and 12.8 -fold, respectively, greater risk of cardiac events (95\% CI 1.2-13.2; 1.2-75.1; 1.799.7). Conclusions. Simple assessments of function and symptoms easily performed at discharge may predict short-term cardiac outcomes in hospitalized HF patients.

\section{Introduction}

Heart failure (HF) is a complex syndrome resulting from structural or functional disorders of the heart that impair ventricular ability to fill with or eject blood [1]. As the final stage of many types of heart disease, HF is a prevalent chronic condition and a major public health issue [2]. The costs of HF are large: it has been called the "most costly cardiovascular disorder" in the USA [3]. The total healthcare expenditure for HF in the USA was 34.4 billion dollars in 2010 [4], and hospitalizations are the top contributor to these costs [2]. HF is the main reason for 6.5 million hospital days annually [1] and the most common condition for hospital admission in people aged 65 and over [2]. The hospital burden of HF is expected to increase with the rapid aging of the USA population: 72 million adults are projected to be over age of 65 by 2030 [5]. Costs will further increase since HF is part of a key quality-related provision in the Affordable Care Act of 2010. This provision decreases hospital reimbursements for 30-day readmission rates not meeting targets for multiple chronic conditions that include HF [6].

Despite current medical treatment, the prognosis for HF is poor, with a 5 -year mortality rate of $45-60 \%$ [2]. In addition to high mortality, disability levels in HF patients are also consistently high. A recent analysis in a national communitybased sample of patients who reported having HF between 2003 and 2008 found that $11 \%$ had disability in activities of daily living (ADLs) and 57\% had mobility disability (much 
difficulty with or inability to walk 2-3 blocks or climb 10 steps). These rates were constant among HF patients in study cohorts back to 1988 [7].

The connection between HF and disability can be understood by the process described in Nagi's model of disability, in which the pathology of a condition leads first to physical signs and symptoms and impairments in body systems and then to functional limitations (physical, psychological, and/or social), and finally to disability [8]. Various measures of physical function have been shown to predict rehospitalization and survival in patients with HF. Decreased mobility in hospitalized patients is associated with adverse outcomes like functional decline $[9,10]$, new institutionalization, and death [9].

Functional decline is both preceded and made worse by signs and symptoms of a condition like HF, according to Nagi's model [8]. HF patients commonly experience a highly variable symptom burden and diminished healthrelated quality of life [11-13]. The symptom burden of HF, including shortness of breath, fatigue, lower extremity edema, and orthopnea [1] is similar to that of advanced cancer [14].

Few researchers have studied whether HF symptoms in an inpatient setting predict postdischarge outpatient outcomes like rehospitalization and mortality. One large retrospective study examined charts of patients hospitalized with decompensated $\mathrm{HF}$ and found that those with fewer documented inhospital symptoms had worse short-term (30 days) mortality [15]. Another prospective cohort study identified symptom clusters in hospitalized HF patients, termed "dyspneic" and "weary," and found that higher levels of distress from these clusters independently predicted one-year cardiac death-free survival and cardiac rehospitalization-free survival, respectively [16]. Although it is known that the timing of assessment of potential risk factors (e.g., symptoms) during the illness trajectory may affect patient outcomes [17], to our knowledge, no studies have examined whether the timing of HF symptom measurement during hospitalization (e.g., at admission or discharge) might predict outcomes such as rehospitalization or survival.

Predictors that help to identify hospitalized HF patients who are at higher risk of adverse cardiac outcomes allow for the development of more effective in-hospital care plans and more targeted interventions to prevent future cardiac events or less aggressive curative efforts if indicated, along with improved discharge coordination and follow-up care. This pilot study was part of a parent study to characterize levels of mobility in hospitalized HF patients using accelerometers. The purpose of this study was to explore multiple measures of function as well as symptoms and to examine related factors that might predict short-term cardiac event-free survival.

1.1. Aims. The specific aims of this pilot study were to examine the (1) function of HF patients at home and between two points in time during hospitalization and (2) symptoms of HF patients at two points in time during hospitalization (3) to determine whether function and/or symptoms predict cardiac event-free survival up to 90 days after hospital discharge. Time points were the study start, which was up to 48 hours after hospital admission, and the study end, which was up to 7 days after hospital admission or the day of discharge if the hospital stay was less than 7 days. Function was measured by NYHA Classification, self-report of home exercise, the Katz Index of independence in ADLs, the short physical function battery, the Karnofsky Performance status Scale, and ambulation. Ambulation was defined as the average daily time spent lying, sitting, and standing or walking. Symptoms were measured by yes/no questions regarding shortness of breath, fatigue, orthopnea, and edema. Cardiac events were defined as rehospitalization attributed to a cardiac cause, heart transplantation, or death.

\section{Materials and Methods}

2.1. Study Design, Setting, and Sample. A convenience sample of 32 patients aged 30 and above was recruited for this prospective cohort study, which took place on two inpatient telemetry units at a large urban academic hospital. The study was approved by the local Institutional Review Board and inclusion criteria included a primary or secondary diagnosis of $\mathrm{HF}$ as determined by medical record review, ability to ambulate with or without an assistive device during the month prior to hospitalization, having a doctors' activity order that allowed the patient to be ambulatory, ability to speak English, and no isolation precautions. Exclusion criteria included dementia as measured by the MiniCog [18] or indicated as severe in the medical record, delirium as measured by the confusion assessment method (CAM) [19], and living in a skilled nursing facility prior to admission.

A trained graduate nursing student conducted all study procedures and data analysis in collaboration with the principal investigator of the parent study. All patients admitted to the study units under either cardiology or medical services within the prior 48 hours were prescreened daily via chart review between April 2010 and February 2011. After prescreening, 103 patients were considered for study approach and 32 were enrolled. Of the 71 patients not enrolled, $46 \%$ had a short length of stay (LOS). Short LOS was defined as a discharge planned on the same or next day according to electronic medical record notes, the bedside nurse, or the medical team. Refusals accounted for $27 \%$ of those not enrolled. The final $27 \%$ were not enrolled for various reasons including having lower extremity skin problems that would interfere with monitor attachment (e.g., leg wraps), speaking a language other than English, cognitive problems, transfer off the unit, or not actually being ambulatory.

Once consented, the Mini-Cog and the CAM were administered to screen for cognitive impairment and delirium, respectively, and the patient was enrolled if eligible. Including the enrollment visit, the study procedure consisted of up to 5 hospital visits and one follow-up phone call after discharge (Table 1).

2.2. Data Collection. Demographic information was collected from medical records at enrollment and confirmed by patient interviews. Clinical information including comorbidities (high risk diagnosis for the elderly score, HRDES 
TABLE 1: Study procedure.

\begin{tabular}{|c|c|c|c|}
\hline Study start—day 1 & Study days $2-4$ & $\begin{array}{l}\text { Study end-day } 5 \\
\text { (or day of discharge) }\end{array}$ & 90 days after discharge \\
\hline Baseline measures & Check-in visits & Final measures & Follow-up call \\
\hline $\begin{array}{l}\text { Demographic: age, gender, race, } \\
\text { marital status } \\
\text { Clinical: vital signs, symptoms, } \\
\text { EF } \\
\text { Functional: home exercise } \\
\text { (self-report), NYHA Class at } \\
\text { home and study start, Katz Index } \\
\text { of ADLs, KPS, SPPB } \\
\text { Ambulation: place accelerometer } \\
\text { monitors on ankle and thigh }\end{array}$ & $\begin{array}{l}\text { Skin: check skin condition under } \\
\text { monitors } \\
\text { Ambulation: move accelerometer } \\
\text { monitors to opposite ankle and } \\
\text { thigh }\end{array}$ & $\begin{array}{l}\text { Clinical: vital signs, symptoms, } \\
\text { HRDES, EF confirmation, receipt } \\
\text { of PT in hospital } \\
\text { Functional: NYHA Class at study } \\
\text { end } \\
\text { Skin: check skin condition under } \\
\text { monitors } \\
\text { Ambulation: remove } \\
\text { accelerometer monitors }\end{array}$ & $\begin{array}{l}\text { Phone call to determine whether } \\
\text { rehospitalization or emergency } \\
\text { room visit occurred }\end{array}$ \\
\hline
\end{tabular}

Abbreviations: ADLs: activities of daily living; EF: ejection fraction; HRDES: high risk diagnosis for the elderly score; KPS: Karnofsky performance status scale; NYHA: New York Heart Association; PT: physical therapy; SPPB: short physical function battery.

[20]) was extracted from medical records when the patient was discharged. Vital signs, NYHA Classification, and symptoms were determined by the study nurse at the beginning and end of the study; NYHA Class was also assessed at home prior to admission (based on self-report of symptoms with activity). Function was assessed at home (self-report of home exercise), at the beginning of the study (Katz Index, KPS, and SPPB), and during the study (ambulation). Both assessments pertaining to the patient at home (NYHA Class and home exercise) were self-reported at the study start in the hospital, based on the patient's recall of his or her functioning prior to admission. The Katz Index and the KPS were nurse-administered questionnaires, while the SPPB was a direct performance measure. Ambulation was measured continuously during the study period via wireless accelerometer monitors.

Telephone follow-up occurred at 90 days following discharge to determine whether patients experienced hospitalizations or emergency department visits. In addition, they were asked about which symptoms led them to seek care. Deaths were confirmed by public death records including internet-accessible obituaries and the Social Security Death Index [21].

2.3. Measures. Refer to Table 2 for a summary of basic information about study measures.

\subsubsection{Function}

NYHA Classification. It was originally developed in 1928 [22]. This well-established instrument describes functional status that ranges from Class I for patients who have no symptoms to Class IV for patients who are symptomatic at rest $[23,24]$. Since it indicates what level of physical activity provokes HF symptoms, NYHA Classification can be viewed as a combined assessment of physical function and symptoms. Classification by NYHA is inherently subjective since it depends on a healthcare provider's interpretation of various levels of physical activity (e.g., what is meant by "ordinary" activity) and whether limitations should be called "slight" or "marked" [22]. This subjectivity has caused some to question the validity of NYHA Classification [25]. However, studies show that NYHA Classification is a valid measure. Although based on subjective assessment by the examiner, the NYHA instrument is valid, correlating well with other functional measures including exercise testing $[17,26-28]$, and tests have found the NYHA classification to be moderately reliable $[25,29]$.

The Katz Index of Independence in ADLs. It measures a person's ability to independently perform ADLs. The Katz Index scores individuals based on six basic ADLs (bathing, dressing, toileting, transferring, continence, and feeding), indicating whether they are dependent (0 points) or independent (1 point) in each function. A score of 6 indicates full function, a score of 4 indicates moderately impaired function, and a score of 2 or less denotes severe functional impairment. Created nearly half a century ago, the Katz Index remains a bestpractice tool for assessing functional status in older adults that has been found to be both reliable and valid in studies in multiple populations including older adults and poststroke patients [30,31].

The Short Physical Performance Battery (SPPB). It is a set of three tests that objectively measure lower extremity physical performance. Patients with scores of 10-12 are classified as having minimal limitations, scores of 7-9 have mild limitations, scores of 4-6 suggest moderate limitations, and scores of 0-3 indicate severe performance limitations. The SPPB was originally developed in the 1990s, when it was tested in a large epidemiological study of nondisabled communitydwelling older adults $[32,33]$. The SPPB was found to be valid and reliable in this study. Other studies have further shown reliability [34], and validity has been demonstrated through correlation with 400-meter walk tests [35].

Ambulation. It was measured via Micro Care Timeliness Monitors, miniature recording 3-axis accelerometer monitors (AugmenTech, Pittsburgh, PA). These monitors were previously tested in a mobility study of hospitalized older adults 
TABle 2: Measures of function and symptoms.

\begin{tabular}{|c|c|c|}
\hline Measure & Type & Brief Description \\
\hline $\begin{array}{l}\text { New York Heart Association (NYHA) } \\
\text { Classification }\end{array}$ & $\begin{array}{l}\text { Function and } \\
\text { Symptoms }\end{array}$ & $\begin{array}{l}\text { Class I: no symptoms or limitations in ordinary physical activity } \\
\text { Class II: mild symptoms and slight limitation in ordinary activity } \\
\text { Class III: symptoms cause marked limitation even during less than } \\
\text { ordinary activity } \\
\text { Class IV: severe limitations; symptoms at rest }\end{array}$ \\
\hline Home exercise & Function & "Do you exercise at home?"-yes/no \\
\hline $\begin{array}{l}\text { Katz Index of independence in activities } \\
\text { of daily living (ADLs) }\end{array}$ & Function & $\begin{array}{l}\text { Ability to perform six ADLs (bathing, dressing, toileting, transferring, } \\
\text { continence, and feeding) independently }\end{array}$ \\
\hline Short physical function battery (SPPB) & Function & $\begin{array}{l}\text { Set of } 3 \text { tests (balance, gait speed, and chair stands) that objectively measure } \\
\text { lower extremity physical function }\end{array}$ \\
\hline Ambulation & Function & $\begin{array}{l}\text { Direct continuous ambulation measurement via wireless accelerometers } \\
\text { attached to lower extremity }\end{array}$ \\
\hline $\begin{array}{l}\text { Karnofsky performance status scale } \\
\text { (KPS) }\end{array}$ & Function & $\begin{array}{l}\text { Rating scale that assesses impact of health condition on ability to work and } \\
\text { care for oneself }\end{array}$ \\
\hline Symptom questions & Symptoms & $\begin{array}{l}\text { Do you have the following symptoms-shortness of breath, fatigue, } \\
\text { orthopnea, or lower extremity edema?-yes/no }\end{array}$ \\
\hline
\end{tabular}

and showed reliability (internal consistency) and validity (comparability to direct patient observation) [36]. In the present study, two accelerometer monitors were programmed and attached to the thigh and ankle of the patient for up to five days, or until discharge. Monitors were removed daily, skin condition was checked, and a new set of monitors was attached to the ankle and thigh on the opposite leg. The HyperTerminal PE program (Hilgraeve Inc., Monroe, MI) was used to program monitors and download data. Gauze pads were used to cushion the monitors against the skin and Tegaderm (3M, St. Paul, MN) secured the monitors.

The Karnofsky Performance Status Scale (KPS). It is a global measurement of function which uses a 100-point rating scale to assess the impact of a health condition on ability to work and care for oneself. The rating scale ranges in increments of 10 from normal function (score of 100) to absence of function (score of 0 , patient deceased), and it was originally developed in 1948 to assess function in cancer patients [37]. Reliability has been tested and validity of the KPS has been established with respect to measures of ADL function and quality of life $[38,39]$.

\subsubsection{Symptoms}

Patient Symptoms. They were assessed via yes/no questions regarding whether or not the patient was experiencing shortness of breath, fatigue, lower extremity edema, or orthopnea.

Self-Report of Home Exercise. It was determined by asking patients whether or not they do exercise at home.

2.4. Statistical Analysis. Demographic and clinical data were analyzed using SPSS Statistics 19 software (IBM Corp., Armonk, NY). Descriptive statistics were used to summarize demographic and clinical data, including function and symptoms. Accelerometer monitor data was processed using Excel (Microsoft, Redmond, WA) and SPSS Statistics 19. Ambulation, defined as average daily time spent in each position (lying, sitting, and standing or walking), was calculated during the study period for each patient. Full analysis of the ambulation data is presented elsewhere. For this analysis, data on function and symptoms were used as independent variables for survival analysis. Survival analyses were completed using a univariate Cox proportional-hazards regression with time to first cardiac event after discharge as the outcome variable. An alpha of 0.05 was used. Hazard ratios and confidence intervals were calculated to identify predictors of cardiac events.

\section{Results}

3.1. Sample Characteristics. Thirty-two patients with a mean age of $58.2 \pm 13.6$ years participated in the study, and $78.1 \%$ were men (Table 3 ). More than half self-identified as white (59.4\%), 31.3\% as African-American, and 9.4\% as Asian or Pacific Islander. Most had a history of hypertension (71.9\%) and mean creatinine on admission was $1.9 \pm 1.7 \mathrm{~g} / \mathrm{dL}$. Most patients had systolic HF: $70.9 \%$ had an EF of less than $40 \%$. The comorbidity score or mean HRDES was $3.3 \pm 1.7$, and the majority $(53.1 \%)$ of patients fell into the intermediate category, with a corresponding $31 \%$ chance of dying in the next year. Length of hospital stay ranged from 1 to 41 days, with a mean of $9.5 \pm 9.9$; median stay was 6.5 days. Two patients $(6.3 \%)$ received an LVAD during the original study admission but after the study was complete; these patients had the longest LOS at 41 days each. Within 90 days after discharge, a total of 3 patients $(9.4 \%)$ died, 1 patient received a heart transplant, and 11 (34.4\%) had cardiac rehospitalization.

3.2. Functional Status. The proportion of patients in each NYHA Class differed across time points (Table 4). Close to half $(46.9 \%)$ of patients reported having experienced symptoms at rest (NYHA Class IV) at home prior to admission. 
TABLE 3: Sociodemographic and clinical characteristics of study patients $(N=32)$.

\begin{tabular}{|c|c|}
\hline Characteristic & Value \\
\hline \multicolumn{2}{|l|}{ Age, years } \\
\hline Mean \pm SD & $58.2 \pm 13.6$ \\
\hline Range & 30-92 \\
\hline \multicolumn{2}{|l|}{ Sex, \% (n) } \\
\hline Male & $78.1(25)$ \\
\hline Female & $21.9(7)$ \\
\hline \multicolumn{2}{|l|}{ Race/ethnicity \% (n) } \\
\hline Caucasian/white & $59.4(19)$ \\
\hline African-American/black & $31.3(10)$ \\
\hline Asian/Pacific Islander & $9.4(3)$ \\
\hline \multicolumn{2}{|l|}{ Marital status, $\%(n)$} \\
\hline Married & $28.1(9)$ \\
\hline Single & $40.6(13)$ \\
\hline Other (widowed/divorced) & $31.3(10)$ \\
\hline \multicolumn{2}{|l|}{ Smoking } \\
\hline History of smoking, $\%(n)$ & $75.0(24)$ \\
\hline Pack years, mean \pm SD & $21.0 \pm 20.7$ \\
\hline History of hypertension, $\%(n)$ & $71.9(23)$ \\
\hline Creatinine on admission $(\mathrm{g} / \mathrm{dL})$, mean $\pm \mathrm{SD}$ & $1.9 \pm 1.7$ \\
\hline \multicolumn{2}{|l|}{ Etiology of heart failure, $\%(n)$} \\
\hline Ischemic & $28.1(9)$ \\
\hline Idiopathic & $65.6(21)$ \\
\hline Unknown/other & $6.3(2)$ \\
\hline Ejection fraction $<40 \%, \%(n)$ & $71.9(23)$ \\
\hline ACEi/ARB use-study end, \% ( $n)$ & $62.5(20)$ \\
\hline Beta blocker use-study end, \% ( $n)$ & $78.1(25)$ \\
\hline \multicolumn{2}{|l|}{ High-risk diagnoses for the elderly scale } \\
\hline Low $(0)=9.5 \%$ chance dying in 1 year & $0(0)$ \\
\hline Intermediate $(1-2)=31 \%$ chance & $53.1(17)$ \\
\hline High risk $(3-5)=46 \%$ chance & $31.3(10)$ \\
\hline Very high risk $(\geq 6)=74 \%$ chance & $15.6(5)$ \\
\hline \multicolumn{2}{|l|}{ Length of hospital stay, days } \\
\hline Mean \pm SD & $9.5 \pm 9.9$ \\
\hline Median & 6.5 \\
\hline Range & $1-41$ \\
\hline Physical therapy-in hospital, \% (n) & $34.4(11)$ \\
\hline LVAD received during study admission, $\%(n)$ & $6.3(2)$ \\
\hline Discharged with physical therapy, occupational therapy, or home health, $\%(n)$ & $40.6(13)$ \\
\hline \multicolumn{2}{|l|}{ Cardiac events-90 days after discharge, $\%(n)$} \\
\hline Cardiac readmission & $34.4(11)$ \\
\hline Heart transplant & $3.1(1)$ \\
\hline Mortality & $9.4(3)$ \\
\hline
\end{tabular}

Abbreviations: ACEi: angiotensin converting enzyme inhibitor; ARB: angiotensin receptor blocker; LVAD: left ventricular assistive device.

By the start of the study, a median of 1.0 days after admission, $18.8 \%$ reported symptoms at rest. Only one patient $(3.1 \%)$ reported symptoms at rest at the study end, a median of 1.0 days prior to discharge.

Most patients (62.5\%) reported exercising at home prior to admission, and the mean Katz Index score was $5.6 \pm 1.1$ (possible 0-6) indicating near independence in ADLs. The mean SPPB score at the study start was $6.4 \pm 3.1$, placing the mean just above the classification of moderate physical function limitation. The largest proportion of patients $(38.7 \%)$ had scores in the moderate functional limitation category (score 4-6). Ambulation measurement during the study 
TABLE 4: Functional status of study patients.

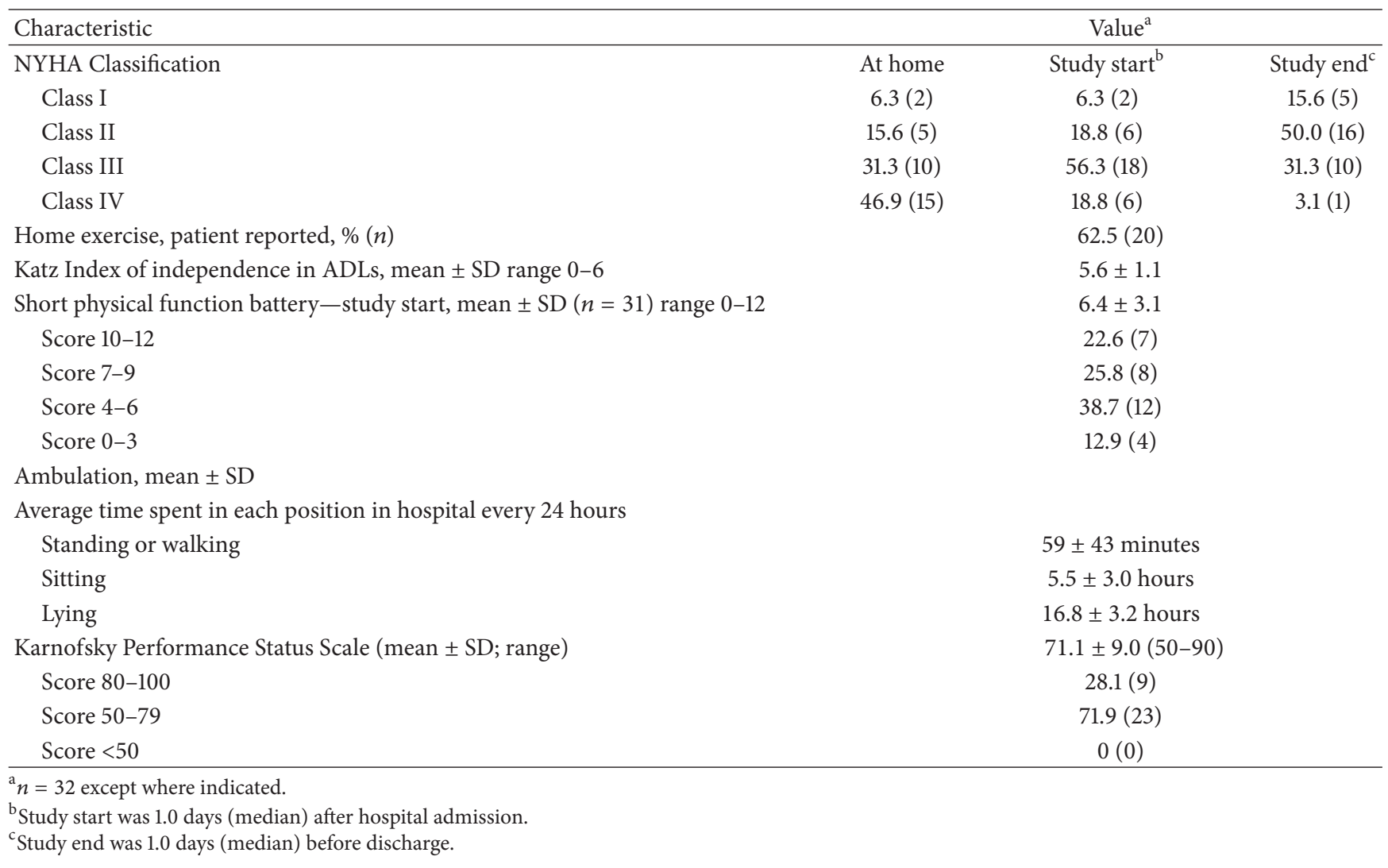

TABLE 5: Number of symptoms reported at beginning and end of study.

\begin{tabular}{lcc}
\hline Statistic & Study start $^{\mathrm{a}}$ & Study end $^{\mathrm{b}}$ \\
\hline$N$ & 32 & 31 \\
Range & $1-4$ & $0-4$ \\
mean \pm SD & $3.3 \pm 1.0$ & $2.2 \pm 1.2$ \\
Median & 4 & 2 \\
\hline
\end{tabular}

${ }^{a}$ Study start was 1.0 days (median) after admission.

${ }^{\mathrm{b}}$ Study end was 1.0 days (median) before discharge.

showed that patients spent an average of $59 \pm 43$ minutes daily standing or walking in the hospital and an average of $16.8 \pm 3.2$ hours lying down.

The mean KPS score was $71.1 \pm 9.0$, with scores ranging from 50 to 90 . Most patients (71.9\%) fell into the middle KPS category of 50-79, indicating that they were unable to work but were able to live at home and care for most needs with varying amounts of assistance. No patients were unable to care for themselves and required the equivalent of hospital care (score $<50$ ), and $28.1 \%$ felt they were able to carry on normal activity without any special care (scores 80-100).

3.3. Symptoms. At the beginning of the study, the median number of symptoms reported was 4.0 out of the four HF symptoms assessed (shortness of breath, fatigue, orthopnea, and edema). The mean was $3.3 \pm 1.0$ symptoms, and all patients reported at least one symptom (Table 5). At the study end, the median number of symptoms reported decreased to 2.0 , the mean was $2.1 \pm 1.3$ symptoms, and $10 \%$ of patients reported no symptoms (Figure 1).

Fatigue was the most prevalent symptom (Figure 2) at both the study start (94\%) and the study end (58\%). Prevalence of SOB decreased the most between the study start and end, from $91 \%$ to $42 \%$, and orthopnea decreased the least, from $63 \%$ to $61 \%$.

3.4. Prediction of Cardiac Event-Free Survival. At least one cardiac event occurred in 13 patients (40.6\%) within 90 days after discharge. Most initial cardiac events $(n=11)$ were cardiac readmissions, including one patient readmitted after the study for heart transplantation. Implantations of LVADs in two patients were not included as cardiac events since they occurred during the same admission as the study. One patient who died within 90 days had a prior cardiac readmission during the follow-up period, so that readmission was analyzed as the first cardiac event. The two additional deaths were analyzed as the first cardiac events.

Of the demographic and clinical characteristics analyzed, two factors were found to be associated with cardiac eventfree survival (Table 6). Results showed that patients were 4.2 times less likely to have a cardiac event if they had a history of hypertension (HR 0.238; 95\% CI 0.08-0.71). Additionally, an increased LOS was associated with a higher risk of cardiac 


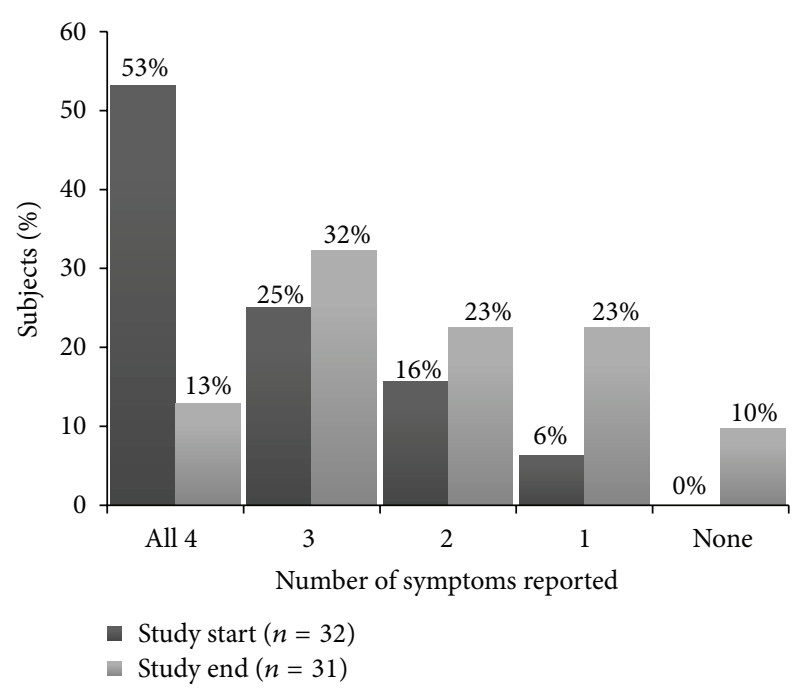

FIgURE 1: Percent of patients reporting different numbers of symptoms at study start and study end. Study start was 1.0 days (median) after admission; study end was 1.0 days (median) before discharge. Symptoms assessed were shortness of breath, fatigue, orthopnea, and edema.

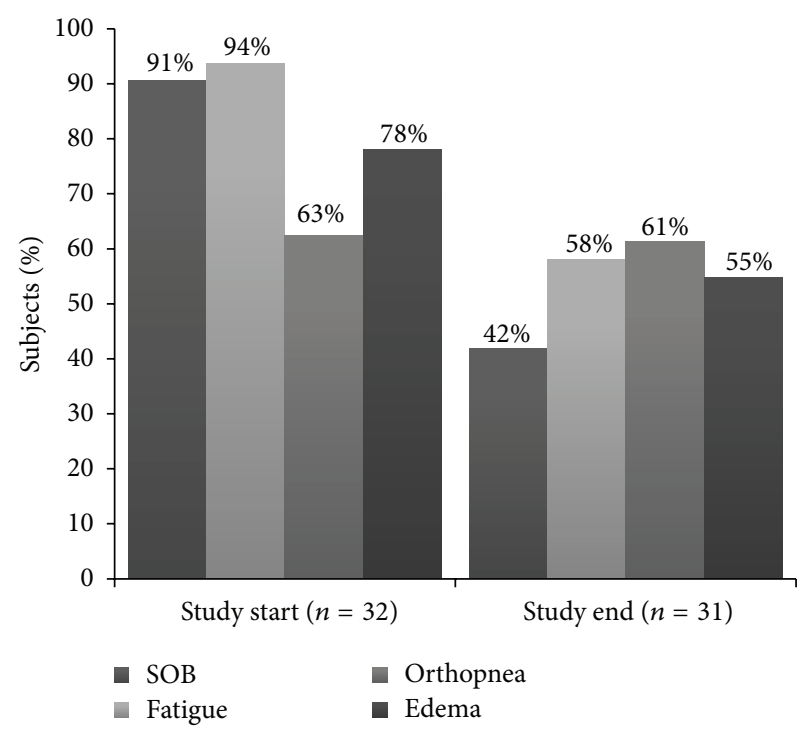

FIGURE 2: Percent of patients reporting individual symptoms common in $\mathrm{HF}$ at the beginning and end of the study. $\mathrm{SOB}=$ shortness of breath. Study start was 1.0 days (median) after admission; study end was 1.0 days (median) before discharge.

events. Results showed an $8.5 \%$ increase in risk with each additional day in the hospital (HR 1.085; 95\% CI 1.03-1.15).

Among the physical function measures, each increase in NYHA Class at the study end was associated with a 3.4-fold higher risk of cardiac events within 90 days (HR 3.404; 95\% CI 1.37-8.46). The sole measure of global function, KPS, was not predictive of cardiac events at the alpha level designated for this study (0.05).

Three of the 4 symptoms reported by patients at the study end (median of 1.0 days before discharge) were significant predictors of cardiac events. Patients reporting SOB at the study end had a 4.0-fold greater risk of cardiac events than those who did not report this symptom (HR 3.962; 95\% CI 1.19-13.22). Risk of cardiac events was 9.7 times higher among patients reporting fatigue at the study end (HR 9.661; 95\% CI 1.24-75.06), and risk of cardiac events was 12.8 times higher among patients who reported orthopnea at study end (HR 12.807; 95\% CI 1.65-99.73). No symptoms reported at study start predicted outcomes, and edema was the only symptom not associated with outcomes at study end.

\section{Discussion}

In this study, we found that higher NYHA Classification and presence of three symptoms (shortness of breath, fatigue, or orthopnea) at the study end (a median of 4.5 days after admission and 1.0 days before discharge) predicted cardiac events in HF patients within 90 days after hospital discharge. These results are unique because they suggest that a quick, simple assessment of function and symptoms that can easily be made at the bedside by physicians or nurses before hospital discharge may be a meaningful way to predict short-term cardiac outcomes in HF patients.

4.1. Function. NYHA Classification at study end (median of 1.0 days before discharge) was found to be predictive of cardiac events in this study, and this is consistent with a recent review that examined risk factors for HF hospitalization. The review noted that NYHA Classification at hospital discharge has been shown to predict both 30-day and 1-year readmissions [17]. Other researchers have also shown that NYHA Classification is associated with mortality. Results from an outpatient study by Devroey and Van Casteren showed that patients who died of HF within 6 months of their diagnosis had a higher NYHA Class at diagnosis than those who did not die [40]. Another study of function in older hospitalized HF patients found that increasing preadmission NYHA Class was associated with greater mortality over a follow-up period of over a year [26]. This is in contrast to results reported here, in which only predischarge, not pre-admission, NYHA Classification predicted outcomes.

The distribution of NYHA Class results from the 32 patients changed from home to the study start and to the study end. Thus, the NYHA Class reflects the dynamic clinical course of $\mathrm{HF}$, from the overall severity of HF exacerbation in the study cohort prior to admission to the symptomatic relief obtained after initial treatment (study start) to further improvements after ongoing treatment (study end). It is common to find NYHA Classification in patient's hospital admission notes, but our results suggest that particular attention should also be paid to assessing and documenting NYHA Classification close to hospital discharge for optimal discharge planning.

Chiarantini and colleagues [26] found that SPPB was related to survival in their cohort study involving older HF patients. A higher SPPB score at discharge conferred greater risk of mortality over a follow-up period of just over one year. These results differ from our study, which 
TABLE 6: Cox univariate predictors of cardiac events within 90 days after discharge.

\begin{tabular}{|c|c|c|c|}
\hline Variable & Hazard ratio (HR) & 95\% confidence interval $(\mathrm{CI})$ & $P$ value $^{\mathrm{a}}$ \\
\hline Age & 0.974 & $0.93-1.02$ & 0.266 \\
\hline Gender & 1.548 & $0.34-6.99$ & 0.570 \\
\hline ACEi or ARB therapy at end of study ${ }^{b}$ & 0.941 & $0.31-2.88$ & 0.916 \\
\hline Beta blocker therapy at end of study & 0.510 & $0.16-1.66$ & 0.264 \\
\hline Ambulation-average daily time spent standing or walking in hospital & 1.006 & $0.99-1.02$ & 0.394 \\
\hline Ambulation-average daily time spent lying down in hospital & 1.067 & $0.90-1.27$ & 0.468 \\
\hline Creatinine & 1.024 & $0.78-1.35$ & 0.869 \\
\hline Home exercise, patient reported & 0.667 & $0.22-1.99$ & 0.467 \\
\hline Physical therapy in hospital & 1.375 & $0.45-4.21$ & 0.576 \\
\hline Discharged with physical or occupational therapy, or home health & 1.327 & $0.45-3.96$ & 0.612 \\
\hline Ejection fraction $<40 \%$ & 2.327 & $0.52-10.52$ & 0.272 \\
\hline History of hypertension & 0.238 & $0.08-0.71$ & 0.010 \\
\hline History of smoking & 1.109 & $0.31-4.03$ & 0.875 \\
\hline Current smoking & 1.484 & $0.70-3.15$ & 0.304 \\
\hline Pack years & 0.999 & $0.97-1.03$ & 0.941 \\
\hline Karnofsky performance status scale category (KPS) & 0.620 & $0.36-1.06$ & 0.082 \\
\hline Katz Index of ADLs—study start & 1.084 & $0.63-1.87$ & 0.770 \\
\hline Length of stay & 1.085 & $1.03-1.15$ & 0.007 \\
\hline NYHA Class-home & 1.607 & $0.79-3.25$ & 0.188 \\
\hline NYHA Class-study start & 1.28 & $0.63-2.59$ & 0.493 \\
\hline NYHA Class—study end & 3.404 & $1.37-8.46$ & 0.008 \\
\hline Shortness of breath-study start ${ }^{\mathrm{c}}$ & 24.449 & $0.15-39302.29$ & 0.396 \\
\hline Shortness of breath-study end & 3.962 & $1.19-13.22$ & 0.025 \\
\hline Fatigue-study start & 22.814 & $0.00-169995.56$ & 0.492 \\
\hline Fatigue-study end & 9.661 & $1.24-75.06$ & 0.030 \\
\hline Orthopnea-study start & 2.462 & $0.68-8.96$ & 0.172 \\
\hline Orthopnea-study end & 12.807 & $1.65-99.73$ & 0.015 \\
\hline Edema—study start & 0.649 & $0.20-2.11$ & 0.472 \\
\hline Edema—study end & 0.660 & $0.21-2.05$ & 0.471 \\
\hline Total number of symptoms-study start & 1.500 & $0.748-3.007$ & 0.254 \\
\hline Total number of symptoms-study end & 2.341 & $1.310-4.182$ & 0.004 \\
\hline SPPB total score-study start & 1.042 & $0.89-1.23$ & 0.618 \\
\hline SPPB balance score-study start & 1.473 & $0.84-2.57$ & 0.173 \\
\hline SPPB gait score-study start & 1.153 & $0.77-1.72$ & 0.488 \\
\hline SPPB chair stand score-study start & 0.869 & $0.57-1.33$ & 0.515 \\
\hline
\end{tabular}

${ }^{a}$ Bold indicates results with $P<0.05$.

${ }^{\mathrm{b}}$ Study end was 1.0 days (median) before discharge.

${ }^{\mathrm{c}}$ Study start was 1.0 days (median) after admission.

found no significant relationship between cardiac events (including mortality) and the SPPB. However, our population was younger, the study follow-up period was shorter, and the SPPB was assessed closer to admission, representing a different time point.

4.2. Symptoms. Among the three symptoms that predicted outcomes, orthopnea conferred the greatest risk for cardiac events, followed by fatigue and shortness of breath. Edema was not predictive at either of the time points measured. These results are similar to those found by investigators in the large European beta-blocker drug trial, COMET (Carvedilol or Metoprolol European Trial). In a secondary analysis of outpatients, investigators reported the patient's NYHA Class and edema and asked patients about breathlessness, fatigue, angina, and orthopnea [41, 42]. The same assessments were conducted at baseline and up to a follow-up period of nearly five years. Univariate analysis showed that only breathlessness, orthopnea, and fatigue were significantly related to the development of worsening $\mathrm{HF}$ and to reduced survival. Assuming that breathlessness is equivalent to shortness of breath, it is striking that these are the same three symptoms found to be significant in univariate analyses in the present study. The COMET analysis $[41,42]$ differed from our study in that it took place in an outpatient setting and examined long-term outcomes. 
Results of our study are congruent with the findings of Song and colleagues [16], who completed a prospective cohort study to identify symptom clusters among inpatients with HF exacerbation and determine their impact on cardiacrelated death and rehospitalization. Symptoms were assessed by questionnaire 1-2 days prior to discharge, and monthly followup lasted one year. Two main physical symptom clusters emerged, termed "dyspneic" (shortness of breath, difficulty of breathing when lying flat, and waking up breathless at night) and "weary" (lack of energy, lack of appetite, and difficulty sleeping). Key results from the study were that a higher level of distress from the weary symptom cluster was an independent predictor of cardiac rehospitalization-free survival, and higher distress from the dyspneic symptom cluster was an independent predictor of cardiac death-free survival. These analyses controlled for clinical variables like age, sex, HF etiology, BMI, EF, and comorbidities, making a strong argument that the symptom experience alone is related to negative outcomes. The "dyspneic" cluster includes two of the three symptoms that were significant in the present study, and they were also measured at a similar time close to discharge. This supports our results and suggests that these same symptoms might also predict longer-term outcomes. Both the symptom cluster study [16] and the COMET analysis $[41,42]$ corroborate our finding that symptoms can predict outcomes in hospitalized HF patients.

4.3. Timing. Our results demonstrate that the timing of assessment is of paramount importance when predicting outcomes. It is known that the point in time when a risk factor is measured during the course of illness (e.g., at diagnosis, hospital admission, or discharge) may affect prediction of outcomes [17]. Hospitalization with acute HF is a time of high risk for patients, in which adverse outcomes are more likely [43]. In this study, function at home prior to admission and function and symptoms at the study start were not related to outcomes, whereas both function (NYHA Classification) and symptoms at the study end were found to predict outcomes. By the end of the study, patients had undergone medical therapy for 4.5 days (median time between admission and study end) and they were 1.0 days (median) from discharge. One possibility is that these patients were inadequately diuresed, leading to continued symptoms close to discharge. Another possibility is that a snapshot of function and symptoms at this time represents the patient's new clinical baseline. For patients who continue to be functionally impaired and have refractory symptoms after 4-5 days of treatment, it may indicate progression of disease that affects prognosis.

4.4. Other Findings. Although it is known from previous studies [44], increased length of stay was associated with increased risk of cardiac events after discharge in this study. Longer LOS may be a marker of severity of illness, comorbidities, or other factors.

History of hypertension (HTN) in this study was found to decrease risk of cardiac events. This could be explained by the greater likelihood of having HF with preserved EF (HFPEF) in patients with longstanding HTN, but having an
EF over $40 \%$ (likely HFPEF) had no effect on outcomes in this study (Table 6). It is known that lower systolic blood pressure increases risk of mortality of HF patients in community and in-hospital settings [43], but this may be a separate phenomenon from history of HTN. In a large survey of hospitalized HF patients in Europe, both a higher admission blood pressure and history of HTN were associated with increased survival at 1 year but not at 3 months [44], as was found in the present study.

4.5. Strengths and Limitations. The important finding of this study was that simple standard measures of function and symptoms can predict short-term outcomes in hospitalized HF patients. Strengths of this pilot study include a theoretically sound and evidence-based premise.

This pilot study has several limitations. A convenience sample was used, the sample size was small, and multivariable analysis was not completed due to the sample size. Also, a formal instrument was not used for assessing symptoms. Many well-characterized instruments exist for measuring multiple symptom dimensions, including prevalence, frequency, and severity, which might provide more insight than yes/no questions alone. While the value of the present study results lies in the simplicity of the predictive assessments, this same feature may also be a limitation. Others have noted that many risk factors can exist in the same patient, so looking at individual factors alone may not provide the most meaningful assessment of risk [43].

In addition, this study included fewer HF patients with shorter stays. Patients who were discharged within the 48-hour enrollment window were often not able to be approached for enrollment. This could mean that the study patients may have been "sicker" or more complicated to manage than their shorter-stay counterparts and therefore may not represent the full range of typical hospitalized HF patients.

Larger studies that include more patients and use formal instruments to measure multiple aspects of symptoms are needed to better characterize the association between symptoms, NYHA Classification, and short-term cardiac outcomes in hospitalized HF patients. Despite these limitations, this small pilot study provides evidence that assessments of basic HF symptoms and functional status before discharge can predict short-term patient outcomes.

\section{Conclusion}

When measured before hospital discharge, NYHA Classification and three of the most common symptoms of HF (orthopnea, fatigue, and shortness of breath) have important independent predictive value for determining risk of cardiac events within 90 days. These simple bedside assessments can be used by physicians or nurses to identify high-risk HF patients, to improve clinical decision-making in the hospital, and to provide insight for discharge planning.

Symptoms and NYHA Classification assessed after admission were not predictive of short-term outcomes in this study, underscoring the importance of the timing of 
assessments used for prognostication. The increased risk of cardiac events in patients with symptoms and higher NYHA Classification close to discharge suggests that symptoms and NYHA Classification could be assessed at this time and may provide outcome information when discharging patients who remain symptomatic after treatment (in this study, treatment duration was a median of 4.5 days). The various pressures to discharge patients quickly must be balanced with the goal of maximizing HF treatment and preventing negative outcomes like cardiac events, including rehospitalization.

\section{Conflict of Interests}

The authors declare that there is no conflict of interests regarding the publication of this paper.

\section{Acknowledgments}

The authors would like to thank Senior Statisticians Dr. Steven Paul and Dr. Bruce Cooper for their support during the statistical analysis of this study. They also thank P. Baiyor for valuable assistance on calculations that facilitated data processing for this study.

\section{References}

[1] H. Hunt, "Focused update incorporated into the ACC/AHA 2005 guidelines for the diagnosis and management of heart failure in adults: a report of the American College of Cardiology Foundation/American Heart Association Task Force on Practice Guidelines: developed in collaboration with the International Society for Heart and Lung Transplantation," Circulation, vol. 119, no. 14, pp. e391-e479, 2010.

[2] A. L. Bui, T. B. Horwich, and G. C. Fonarow, "Epidemiology and risk profile of heart failure," Nature Reviews Cardiology, vol. 8, no. 1, pp. 30-41, 2011.

[3] S. Thomas and M. W. Rich, "Epidemiology, pathophysiology, and prognosis of heart failure in the elderly," Clinics in Geriatric Medicine, vol. 23, no. 1, pp. 1-10, 2007.

[4] P. A. Heidenreich, J. G. Trogdon, O. A. Khavjou et al., "Forecasting the future of cardiovascular disease in the United States: a policy statement from the American Heart Association," Circulation, vol. 123, no. 8, pp. 933-944, 2011.

[5] W. He, M. Sengupta, V. A. Velkoff, and K. A. DeBarros, U.S. Census Bureau Current Population Reports, 65+ in the United States: 2005, U.S. Government Printing Office, Washington, DC, USA, 2005, http://www.census.gov/prod/2006pubs/p23-209.pdf.

[6] C. Bielaszka-DuVernay, "Health policy brief: improving quality and safety," Health Affairs, vol. 32, no. 1, pp. 1-7, 2011.

[7] C. Y. Wong, S. I. Chaudhry, M. M. Desai, and H. M. Krumholz, "Trends in comorbidity, disability, and polypharmacy in heart failure," American Journal of Medicine, vol. 124, no. 2, pp. 136143, 2011.

[8] A. A. Guccione and D. A. Scalzitti, "Examination of functional status and activity level," in Physical Rehabilitation, S. B. O'Sullivan and T. J. Schmitz, Eds., F.A. Davis Company, Philadelphia, Pa, USA, 5th edition, 2007.

[9] C. J. Brown, R. J. Friedkin, and S. K. Inouye, "Prevalence and outcomes of low mobility in hospitalized older patients," Journal of the American Geriatrics Society, vol. 52, no. 8, pp. 1263-1270, 2004.

[10] A. Zisberg, E. Shadmi, G. Sinoff, N. Gur-Yaish, E. Srulovici, and H. Admi, "Low mobility during hospitalization and functional decline in older adults," Journal of the American Geriatrics Society, vol. 59, no. 2, pp. 266-273, 2011.

[11] D. B. Bekelman, E. P. Havranek, D. M. Becker et al., "Symptoms, depression, and quality of life in patients with heart failure," Journal of Cardiac Failure, vol. 13, no. 8, pp. 643-648, 2007.

[12] C. D. Blinderman, P. Homel, J. A. Billings, R. K. Portenoy, and S. L. Tennstedt, "Symptom distress and quality of life in patients with advanced congestive heart failure," Journal of Pain and Symptom Management, vol. 35, no. 6, pp. 594-603, 2008.

[13] C. H. Zambroski, D. K. Moser, G. Bhat, and C. Ziegler, "Impact of symptom prevalence and symptom burden on quality of life in patients with heart failure," European Journal of Cardiovascular Nursing, vol. 4, no. 3, pp. 198-206, 2005.

[14] D. B. Bekelman, J. S. Rumsfeld, E. P. Havranek et al., "Symptom burden, depression, and spiritual well-being: a comparison of heart failure and advanced cancer patients," Journal of General Internal Medicine, vol. 24, no. 5, pp. 592-598, 2009.

[15] R. J. Goldberg, F. A. Spencer, M. Szklo-Coxe et al., "Symptom presentation in patients hospitalized with acute heart failure," Clinical Cardiology, vol. 33, no. 6, pp. E73-E80, 2010.

[16] E. K. Song, D. K. Moser, M. K. Rayens, and T. A. Lennie, "Symptom clusters predict event-free survival in patients with heart failure," Journal of Cardiovascular Nursing, vol. 25, no. 4, pp. 284-291, 2010.

[17] G. Giamouzis, A. Kalogeropoulos, V. Georgiopoulou et al., "Hospitalization epidemic in patients with heart failure: risk factors, risk prediction, knowledge gaps, and future directions," Journal of Cardiac Failure, vol. 17, no. 1, pp. 54-75, 2011.

[18] D. M. Carolan Doerflinger, "How to try this: the mini-cog," The American Journal of Nursing, vol. 107, no. 12, pp. 62-72, 2007.

[19] C. M. Waszynski, "How to try this: detecting delirium," The American Journal of Nursing, vol. 107, no. 12, pp. 50-5960, 2007.

[20] M. M. Desai, S. T. Bogardus Jr., C. S. Williams, G. Vitagliano, and S. K. Inouye, "Development and validation of a riskadjustment index for older patients: the high-risk diagnoses for the elderly scale," Journal of the American Geriatrics Society, vol. 50, no. 3, pp. 474-481, 2002.

[21] Ancestry.com. Social Security Death Index [database on-line]. Ancestry.com Operations Inc., Provo, Utah, USA, 2011.

[22] M. Severo, R. Gaio, P. Lourenço, M. Alvelos, P. Bettencourt, and A. Azevedo, "Indirect calibration between clinical observersapplication to the New York Heart Association functional classification system," BMC Research Notes, vol. 4, article 276, 2011.

[23] American Heart Association, "Classes of Heart Failure. American Heart Association web site," 2011, http://www.heart.org/HEARTORG/Conditions/HeartFailure/ AboutHeartFailure/Classes-of-Heart-Failure_UCM_306328 Article.jsp.

[24] R. F. LeBlond, R. L. DeGowin, and D. D. Brown, "The chest: chest wall, pulmonary, and cardiovascular systems, the breasts," in DeGowin's Diagnostic Examination, R. F. LeBlond, R. L. DeGowin, and D. D. Brown, Eds., chapter 8, 9th edition, 2009.

[25] C. Raphael, C. Briscoe, J. Davies et al., "Limitations of the New York Heart Association functional classification system and self-reported walking distances in chronic heart failure," Heart, vol. 93, no. 4, pp. 476-482, 2007. 
[26] D. Chiarantini, S. Volpato, F. Sioulis et al., "Lower extremity performance measures predict long-term prognosis in older patients hospitalized for heart failure," Journal of Cardiac Failure, vol. 16, no. 5, pp. 390-395, 2010.

[27] J. A. Bennett, B. Riegel, V. Bittner, and J. Nichols, "Validity and reliability of the NYHA classes for measuring research outcomes in patients with cardiac disease," Heart and Lung, vol. 31, no. 4, pp. 262-270, 2002.

[28] L. V. Athanasopoulos, A. Dritsas, H. A. Doll, and D. V. Cokkinos, "Comparative value of NYHA functional class and quality-of-life questionnaire scores in assessing heart failure," Journal of Cardiopulmonary Rehabilitation and Prevention, vol. 30, no. 2, pp. 101-105, 2010.

[29] L. Goldman, B. Hashimoto, E. F. Cook, and A. Loscalzo, "Comparative reproducibility and validity of systems for assessing cardiovascular functional class: advantages of a new specific activity scale," Circulation, vol. 64, no. 6, pp. 1227-1234, 1981.

[30] A. Marengoni, E. von Strauss, D. Rizzuto, B. Winblad, and L. Fratiglioni, "The impact of chronic multimorbidity and disability on functional decline and survival in elderly persons. A community-based, longitudinal study," Journal of Internal Medicine, vol. 265, no. 2, pp. 288-295, 2009.

[31] M. Wallace and M. Shelkey, "Monitoring functional status in hospitalized older adults," The American Journal of Nursing, vol. 108, no. 4, pp. 64-72, 2008.

[32] J. M. Guralnik, E. M. Simonsick, L. Ferrucci et al., "A short physical performance battery assessing lower extremity function: association with self-reported disability and prediction of mortality and nursing home admission," Journals of Gerontology, vol. 49, no. 2, pp. M85-M94, 1994.

[33] J. M. Guralnik, L. Ferrucci, E. M. Simonsick, M. E. Salive, and R. B. Wallace, "Lower-extremity function in persons over the age of 70 years as a predictor of subsequent disability," The New England Journal of Medicine, vol. 332, no. 9, pp. 556-561, 1995.

[34] S. Studenski, S. Perera, D. Wallace et al., "Physical performance measures in the clinical setting," Journal of the American Geriatrics Society, vol. 51, no. 3, pp. 314-322, 2003.

[35] S. P. Sayers, J. M. Guralnik, A. B. Newman, J. S. Brach, and R. A. Fielding, "Concordance and discordance between two measures of lower extremity function: 400 meter self-paced walk and SPPB," Aging, vol. 18, no. 2, pp. 100-106, 2006.

[36] C. J. Brown, D. L. Roth, and R. M. Allman, "Validation of use of wireless monitors to measure levels of mobility during hospitalization," Journal of Rehabilitation Research and Development, vol. 45, no. 4, pp. 551-558, 2008.

[37] J. W. Yates and W. B. Ershler, "Have we moved beyond the Karnofsky score?” Clinical Oncology Alert, vol. 26, no. 11, pp. 83-84, 2010.

[38] V. Mor, L. Laliberte, J. N. Morris, and M. Wiemann, "The Karnofsky Performance Status Scale. An examination of its reliability and validity in a research setting," Cancer, vol. 53, no. 9, pp. 2002-2007, 1984.

[39] B. J. Liem, J. M. Holland, M. Y. Kang, S. C. Hoffelt, and C. M. Marquez, "Karnofsky Performance Status assessment: resident versus attending," Journal of Cancer Education, vol. 17, no. 3, pp. 138-141, 2002.

[40] D. Devroey and V. van Casteren, "Symptoms and clinical signs associated with hospital admission and mortality for heart failure," Central European Journal of Public Health, vol. 18, no. 4, pp. 209-214, 2010.

[41] I. Ekman, J. G. F. Cleland, K. Swedberg, A. Charlesworth, M. Metra, and P. A. Poole-Wilson, "Symptoms in patients with heart failure are prognostic predictors: insights from COMET," Journal of Cardiac Failure, vol. 11, no. 4, pp. 288-292, 2005.

[42] I. Ekman, J. G. F. Cleland, K. Swedberg, A. Charlesworth, M. Metra, and P. A. Poole-Wilson, "Erratum in: Symptoms in patients with heart failure are prognostic predictors: insights from COMET," Journal of Cardiac Failure, vol. 11, no. 5, p. 404, 2005.

[43] G. C. Fonarow, "Epidemiology and risk stratification in acute heart failure," American Heart Journal, vol. 155, no. 2, pp. 200 207, 2008.

[44] V.-P. Harjola, F. Follath, M. S. Nieminen et al., "Characteristics, outcomes, and predictors of mortality at 3 months and 1 year in patients hospitalized for acute heart failure," European Journal of Heart Failure, vol. 12, no. 3, pp. 239-248, 2010. 


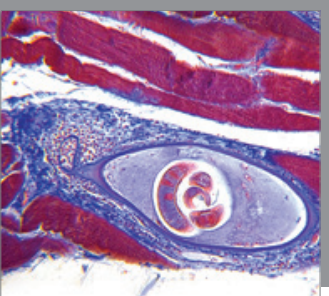

Gastroenterology

Research and Practice
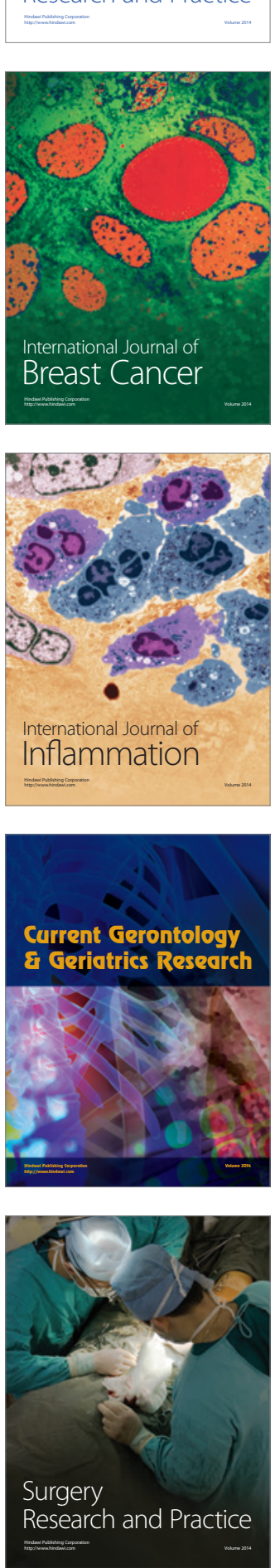

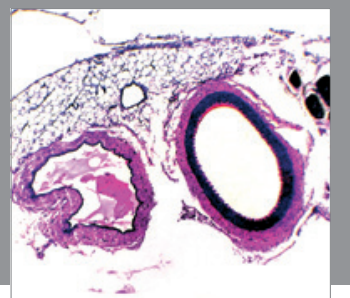

International Journal of Hypertension
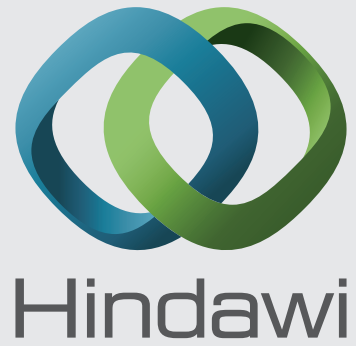

Submit your manuscripts at http://www.hindawi.com
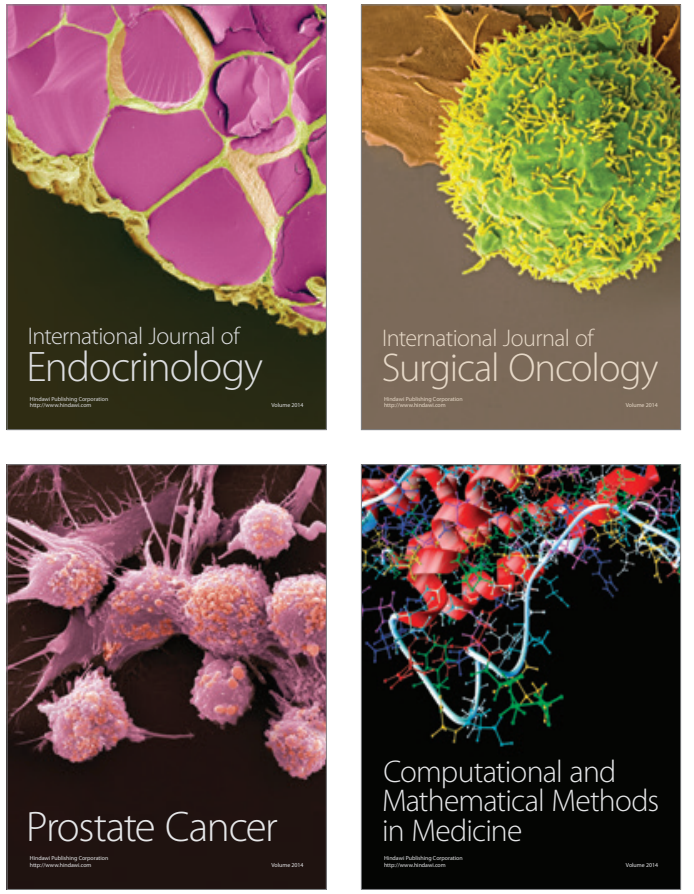
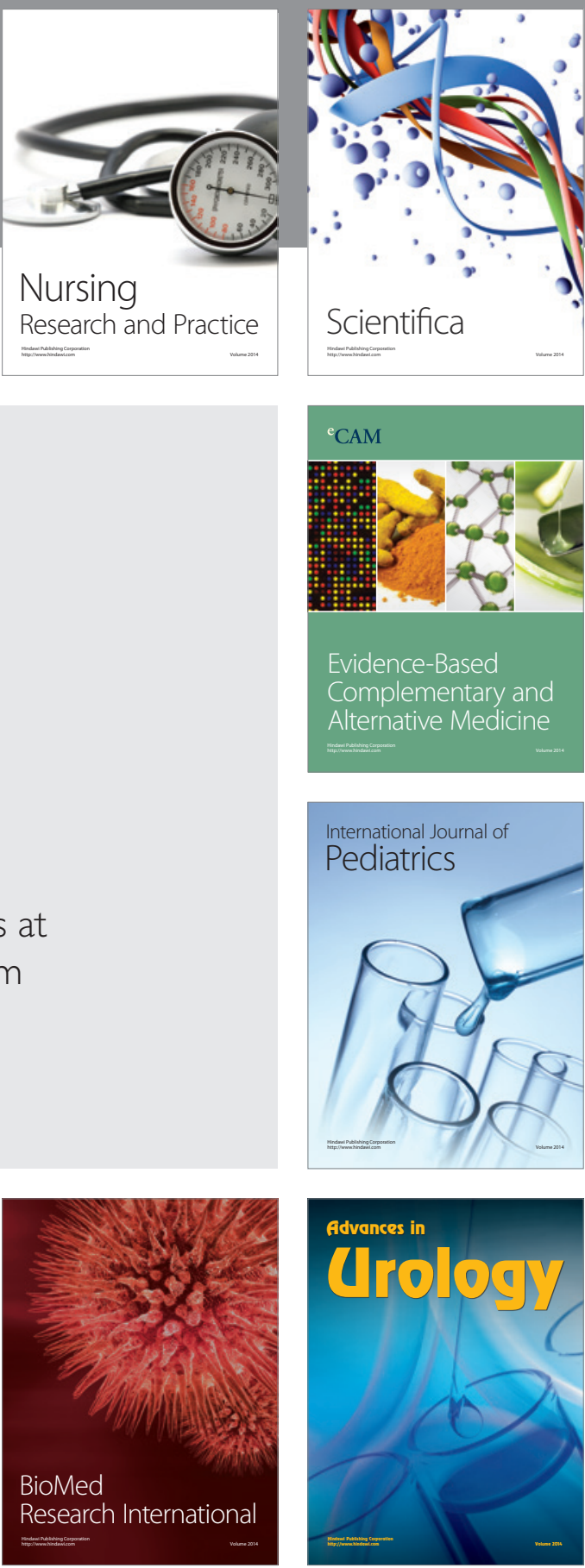

Nursing

Research and Practice

Scientifica

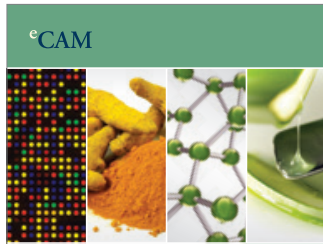

Evidence-Based

Complementary and Alternative Medicine
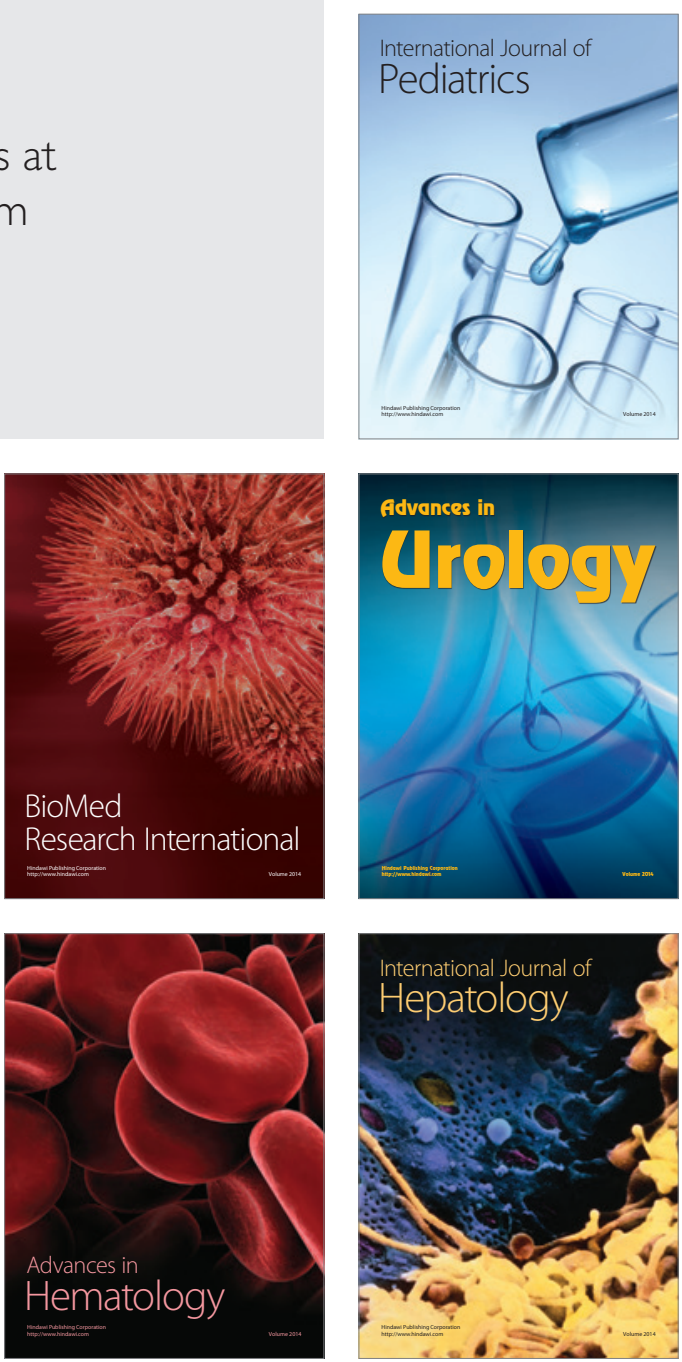